BULLETIN Bulletin hispanique

HISPANIQUE Université Michel de Montaigne Bordeaux

120-1 | 2018

Varia

Miguel de Unamuno, Epistolario I (1880-1899). Introducción, edición y notas de Colette y JeanClaude RABATÉ

Salamanca, Ediciones Universidad de Salamanca, 2017

Jean-François Botrel

\title{
OpenEdition
}

Journals

Edición electrónica

URL: https://journals.openedition.org/bulletinhispanique/6292

DOI: 10.4000/bulletinhispanique.6292

ISSN: 1775-3821

Editor

Presses universitaires de Bordeaux

Edición impresa

Fecha de publicación: 30 junio 2018

Paginación: 366-370

ISBN: 979-10-300-0298-0

ISSN: 0007-4640

Referencia electrónica

Jean-François Botrel, «Miguel de Unamuno, Epistolario I (1880-1899). Introducción, edición y notas de Colette y Jean-Claude RABATÉ», Bulletin hispanique [En línea], 120-1 | 2018, Publicado el 30 junio 2018, consultado el 08 enero 2022. URL: http://journals.openedition.org/bulletinhispanique/6292 ; DOI: https://doi.org/10.4000/bulletinhispanique.6292

Este documento fue generado automáticamente el 8 enero 2022.

Tous droits réservés 


\section{Miguel de Unamuno, Epistolario I (1880-1899). Introducción, edición y notas de Colette y Jean-Claude RABATÉ}

Salamanca, Ediciones Universidad de Salamanca, 2017

Jean-François Botrel

\section{REFERENCIA}

Miguel de Unamuno, Epistolario I (1880-1899). Introducción, edición y notas de Colette y Jean-Claude RABATÉ, Salamanca, Ediciones Universidad de Salamanca, 2017, 1112 p. (Biblioteca Unamuno, 46).

1 En 1916, Emilia Pardo Bazán écrivait à Miguel de Unamuno : «Es usted un Sévigné. Lo mejor de cuanto usted hace es lo epistolar. Y no crea que esto es un grano de anís. Ya van quedando pocas gentes que sepan tornear (sin proponérselo, sin afectación) las cartas y los billetes».

2 La lecture de ce premier tome de l'Epistolario d'Unamuno qui offre, chronologiquement ordonnées, 303 lettres sur les 2500 et quelques qui devraient ainsi être réunies, permettra à chacun de vérifier l'exactitude de l'appréciation s'agissant du jeune Unamuno (il a 35 ans en 1899), déjà affecté par ce que lui-même qualifiera rapidement d'epistolomanía.

3 En l'occurrence, il s'agit moins de la fréquence de l'activité et du nombre de lettres que de son intensité et de la diversité de la pratique, et surtout du statut de l'épistolographie dans les rapports d'Unamuno à l'écriture.

4 Avec une moyenne de 27 lettres par an (44 entre 1924 et 1930), on n'a pas, de prime abord, l'impression qu'Unamuno ait eu une pratique assidue de l'écriture épistolaire, 
mais il faut rappeler que beaucoup de ses lettres ont disparu (c'est le cas, par exemple, de celles à Lázaro Galdiano, le directeur de La España Moderna, qui lui en a pourtant adressé 40 à partir de 1893) et que de nombreuses lettres d'Unamuno font référence à des échanges antérieurs dont on n'a pas conservé de traces, comme ceux avec Narcís oller. Par ailleurs, il s'agit rarement de billets ou cartes de visite, mais en général de lettres longues et fort substantielles : une moyenne de 8500 signes (espaces comprises) entre 1880 et 1899. Il suffit de considérer les lettres adressées à Pedro de Múgica (pas loin d'une par mois entre 1890 et 1895 et toutes particulièrement nourries), pour se convaincre de l'intensité que peut revêtir chez Unamuno la pratique épistolaire.

Cette pratique concerne évidemment le for privé les lettres intimes à sa famille - celle, inachevée $\left(n^{\circ} 120\right)$, à sa mère à propos de son adhésion au Partido Socialista Obrero Español est particulièrement intéressante -, les lettres d'affaires dites "pragmatiques ", comme celles à Fernández Villegas, lié à La Época et à La España Moderna, qui presque toujours prennent cependant un tour plus profond, auxquelles on peut ajouter les lettres administratives. Mais elles concernent tout autant l'espace public, avec toutes les épitres (epístolas), mais aussi les lettres ouvertes que les éditeurs ont très justement décidé de recueillir. Elles constituent «una creación híbrida, coyuntural, y oscilan según las circunstancias y los interlocutores entre la libre conversación, la diatriba, la demostración, transformándose a veces en ensayos » (p. 32). Il y a même des lettres fictives, comme celle adressée à lui-même ( $\left.n^{\circ} 259\right)$ signée d'un certain Crisógono et qu'Unamuno «sin comentario alguno entreg(a) al público, por si alguien gusta de hacérselo a su sabor».

6 Chez Unamuno, encore plus que chez d'autres sans doute, écrire (des lettres) est comme une nécessité interne : il s'agit d'«echar fuera lo que [le] estorba adentro» (p. 23), de «desahogos» (p. 24), et parfois de sorte d'examens de conscience ou de confessions à voix haute. Et il se montre d'ailleurs assez peu préoccupé de conserver un caractère confidentiel à sa correspondance la plus privée qui est rapidement vouée à circuler. À certains moments, comme durant son exil forcé à Fuerteventura, le trop grand nombre de lettres à écrire l'amènera à s'efforcer de «distribuirlas a los puntos más distantes entre sí $y$ de modo que una sirva para muchos» (Cartas del destierro. Entre el odio y el amor (1924-1930). Edición de Colette y Jean-Claude Rabaté, Salamanca, Ediciones Universidad de Salamanca, 2012, p. 464). Chez Unamuno, la frontière entre le privé et le public est donc ténue et il en va de même s'agissant de la lettre et de l'article de presse : en témoigne évidemment le fréquent recours à la lettre ouverte ou à la lettre au directeur (du Noticiero Bilbaíno, de La Democracia, du Diario de Bilbao, etc.). C'est d'ailleurs souvent la presse qui est à l'origine de l'initiative épistolaire: c'est la lecture d'une «Revista crítica» de Clarín qui est le prétexte pour une première lettre commençant par un très conventionnel «Muy señor mío», et la réception du premier numéro de la Revista Crítica de Historia y Literatura Españolas donne le départ de la correspondance avec Altamira, tout comme la publication d'Idearium español incite Unamuno à entrer en contact avec Ganivet pour une intense correspondance (12 lettres entre juin et novembre 1898, y compris une lettre post mortem adressée à Ángel Ganivet «donde estuviere» ( $\left.\mathrm{n}^{\circ} 249\right)$, restée à l'état de projet); c'est un article d'Unamuno qui amène Galdós ou Rubén Darío à lui écrire, etc.

7 Manifestement Unamuno ne fait pas de vrai départ entre l'écriture épistolaire et les autres formes d'écriture, et il est fascinant de voir comment au sein d'une même lettre, sans crier gare, l'épistolographe se transforme en publiciste ou essayiste. On se 
demande même souvent quelle différence il y a au total - sauf dans la forme écrite ou orale - entre les épitres d'Unamuno et ses « sermons laïques ».

Ce qui ne l'empêche pas, le cas échéant, de distinguer entre la pratique privée de la lettre et le caractère public de l'article : c'est ce qu'il fait observer à Clarín dont il reprend très doctement une erreur à propos de l'étymologie de adolescencia : «creo deber hacerlo privadamente» (p. 499); "que no ha sido mi fin darle una lección ni mucho menos le probará el haberlo hecho privadamente» (p.500), insiste-t-il. Une sorte de faveur faite à celui dont les critiques « grammaticales » étaient alors si redoutées.

Tout cela est synthétiquement exprimé dans la citation d'Unamuno mise en exergue (p. 9): «Para excitarme a pensar cosa pública, necesito comunicarme; pienso en voz alta; de mis cartas privadas salen artículos; mis mejores frases públicas se me han ocurrido, de pronto, en conversación o en correspondencia privada», et parfaitement mis en lumière dans la substantielle introduction à ce premier tome de l'Epistolario dont les pages 13 à 38 sont un véritable petit essai sur le genre épistolaire unamunien et valent pour l'ensemble de la collection à venir. Mais l'introduction s'attache aussi à commenter ce dont ces lettres de jeunesse d'Unamuno rendent compte (cf. "Síntesis de las cartas de juventud", pp.39-79). Y résonnent, en effet, beaucoup de thèmes d'actualité précisément analysés et éclairés (Cuba et les Philippines, l'anarchisme et les procès de Montjuic), mais aussi des sujets plus personnels (l'adhésion au Parti socialiste, par exemple), Salamanque, Bilbao ou la Catalogne et, bien sûr, les "coulisses de la création » (notamment celles de Paz en la guerra) ou la conquête de collaborations dans la presse sud-américaine, sans oublier les questions philologiques omniprésentes dans les lettres à Pedro de Múgica et l'inscription décidée d'Unamuno dans un espace déjà parfaitement européen.

10 Mais chacun a le droit de tirer de ce riche corpus les informations qu'il souhaite: les lettres publiées dans El Nervión en 1893 ont, par exemple, pu servir à Carlos Serrano pour étudier la polémique, à propos de l'ensanche de Bilbao, entre Exóristo (Unamuno) et X (Pablo de Alzola) en 1893 (Miguel de Unamuno. Entre histoire et littérature, Paris, Presses de la Sorbonne Nouvelle, 2004, pp. 71-83), et Colette et Jean-Claude Rabaté y ont puisé de précieux et abondants éléments pour leur grande biographie d'Unamuno (Miguel de Unamuno. Biografía, Madrid, Taurus, 2009). De façon plus anecdotique ou intéressée, on pourra aussi, tout simplement, prendre note de la médiocre estime d'Unamuno pour Palacio Valdés ou, au contraire, de son admiration pour la poésie «láctea» de Vicente Medina ou encore pour Pérez Galdós qui, «sin perdón sea dicho», écrit Unamuno (p. 447), «se caga en la corrección» (de la langue), et est «quien mejor refleja en sus libros el castellano real y efectivo» (p. 507). Tel passage d'une lettre à Ortega Munilla, le directeur de Los Lunes de El Imparcial à qui Unamuno, en quête de collaborations dans la presse, adresse spontanément un récit intitulé «El poema vivo del amor» révèle quelqu'un de très ouvert et assez accommodant a priori: «es mi mayor empeño ser flexible y no encerrarme nunca en mi propio criterio o en mi gusto personal», écrit-il le 10 avril 1899 (p. 875).

11 Là ne s'arrête pas, cependant, l'intérêt que représentent ces lettres : il s'agit aussi d'une invitation à réfléchir sur la spécificité de l'écriture épistolaire, qu'elle soit ou non destinée à la publication; une spécificité sans doute à rechercher dans la relation particulière avec lui-même que suppose chez Unamuno le «monodiálogo», mais aussi dans le dialogue qu'il souhaite établir avec le destinataire sous forme de correspondance ; cette façon de penser à voix haute devant celui qui ne peut que le lire 
en silence et qui peut-être lui répondra; un destinataire incarné que quelques documents iconographiques auraient peut-être pu donner à voir.

En ce sens tous les epistolarios - correspondances à une seule voix - ont cela de frustrant qu'ils ne permettent que très indirectement d'apprécier le rôle de l'autre dans la démarche épistolaire, bien que les quelque 20000 "documents » conservés à la Casa Museo Unamuno à Salamanque et parfois publiés soient là qui offrent au lecteur des lettres d'Unamuno la possibilité d'établir ou rétablir ces éventuels et inégaux dialogues à distance.

La matérialité même de la lettre et de son écriture (un ou deux facsimilés de lettres permettraient d'en rendre compte) traduisent bien ce parti-pris et cette démarche monodialogique ou dialogique et pédagogique qui anime l'épistolographe, les insistances et les inflexions de la voix, si importante dans cette conversation à distance, signifiées par le soulignement de phrases ou de mots qui posent à l'éditeur la question du choix de leur traduction typographique : italique, caractères gras ou soulignage qui est la solution adoptée dans la présente édition.

S'agissant précisément des choix éditoriaux (explicités pp. 87-96), on ne peut que louer leur honnêteté, leur rigueur et leur efficacité : respecter les transcriptions existantes (la plupart des lettres ont déjà fait l'objet de publications éparses), les compléter ou les améliorer lorsque cela est possible (les passages rétablis sont imprimés avec une très belle encre rouge) et en permettre une correcte compréhension, tout cela garantit à une édition qui n'est pas paléographique, à la fois la lisibilité et la maniabilité à toutes fins. On appréciera, particulièrement, pour tout le travail qu'il suppose et pour sa totale efficacité, l'index-dictionnaire (pp. 1009-1095) qui permet d'identifier et de repérer, sans avoir à surcharger les lettres de notes, les quelque 1200 personnes et la centaine de publications périodiques qui, dès cette période, peuplent le déjà vaste monde du jeune Miguel de Unamuno.

15 Si Unamuno s'est défendu d'écrire des lettres pour la postérité, il a donné suffisamment d'indices ou de preuves de ce qu'il considérait son activité épistolaire comme une partie indissociable de son activité d'écrivain et publiciste et on peut considérer qu'avec l'édition complète, ordonnée et éclairée de cette partie de son œuvre, on répond de toute évidence, non seulement à un souhait implicite mais aussi à une nécessité pour l'histoire littéraire et intellectuelle. Par ce travail de collection et d'édition, c'est toute la partie souvent cachée de l'œuvre d'un écrivain et d'un intellectuel qui est rendue manifeste mais c'est aussi la confirmation, particulièrement pertinente dans le cas d'Unamuno, qu'on se saurait dissocier l'œuvre épistolaire de l'œuvre totale.

Pour celles ou ceux qui prévoient d'acquérir la collection des 2200 et quelques lettres restantes - une entreprise de titans !-, il faut penser à réserver un demi-mètre linéaire dans leur bibliothèque. 


\section{AUTORES}

JEAN-FRANÇOIS BOTREL

Université Rennes-2 